For more than a decade, US leaders have been trying to move beyond that troubled past and recruit minorities into science and engineering. There are strong moral arguments for doing so. But in times of massive budgetary shortfalls, morals do not guarantee funds. Congress and the public should recognize the powerful practical reasons to support programmes that aim to raise the numbers of minorities in science.

A key issue is that of numbers. There is concern in the United States about the shrinking proportion of home-grown scientists. Foreign-born students, particularly from China and India, account for almost all of the growth in the number of science doctoral degrees granted in America. And many then take their skills back home. Minorities make up a growing share of the US population and represent a relatively untapped pool from which to draw the next generation of scientists.

They also bring fresh ideas to research. This sometimes results in the pursual of topics that can help specific communities but have not managed to capture the attention of mainstream researchers. An example of which is Katie McDonald, who embarked on a research project as an American Indian student at a tribal college in Montana. She found higher-than-expected levels of mercury in local fish and has helped her own tribe to avoid health problems (see page 25).

Bringing more diversity into the ranks of researchers will help to overcome the lingering suspicion of science that persists in some minority communities. In doing so, it will encourage members of the public to accept the products of research, whether they are government health recommendations or reports about the changing climate. Without that kind of trust, researchers could see their work ignored by segments of the population.

For these and other reasons, the US government has poured

substantial funds into pulling more underrepresented minorities into science. The National Science Foundation spent more than $\$ 110$ million on this in 2010, and other agencies, such as the National Institutes of Health, NASA and the US Department of Education, also have programmes to boost minority participation in science.

These initiatives still have a long way to go. The National Research Council (NRC) reported last year that underrepresented minori-

"Minorities represent a relatively untapped pool from which to draw the next generation of scientists." ties made up $28 \%$ of the US population in 2006 but accounted for only $9 \%$ of collegeeducated Americans in the science and engineering workforce.

And in some cases, the numbers are proving hard to move. In 2008, American Indians comprised just $0.7 \%$ of the bachelor's degrees awarded in science and engineering - a proportion that is unchanged since 2000 . Science bachelor's degrees earned by black students has also stayed constant at $8.3 \%$. For doctoral degrees, the figures are even starker. American Indians, who represent $1 \%$ of the population, earn only $0.3 \%$ of the PhDs in science and engineering. Black people make up $13 \%$ of the US population but accounted for just $3 \%$ of the doctoral degrees awarded in 2008 in these fields.

The problem creates a vicious cycle. Similar proportions of minority and white students enter university intending to study science. However, the completion rate for minorities is lower. Many factors contribute to this gap, according to the NRC, but one remains the poor diversity of university faculty members and the scarcity of role models in science for students from minority groups.

\section{Dark rumblings}

\section{The Large Hadron Collider is stirring up trouble, and that's good news for science.}

\section{$\mathrm{I}$} n the 1860s, physics looked beautiful. The Scottish physicist James Clerk Maxwell had just published a series of papers that unified electricity, magnetism and light into a theory that could be expressed in a few equations. In doing so, he settled a long-running debate over whether light was a continuous wave of energy or a spray of tiny particles. It was, to anyone who understood Maxwell's work, quite obviously a wave. That raised a question, although it seemed to be more of a niggling detail to Maxwell's devotees: like water waves or sound, the new, electromagnetic light waves should need a medium through which to travel. If Maxwell was right, what did it look like?

So began the search for the notorious ether. In one spectacular experiment in 1887, Albert Michelson and Edward Morley designed and built a prototype interferometer to measure the speed of light at different points in Earth's orbit and showed that the speed was constant - impossible if light and Earth were flowing through an unseen liquid. Contrary to all their expectations, the ether wasn't there.

There are some parallels between physics then and physics now. Like the 1860s, the 1960s saw an incredible unification of modern physical theories. This time, the standard model of particle physics took Maxwell's electromagnetic force and wove it with the strong and weak nuclear forces. According to the theory, at sufficiently high energies the weak and electromagnetic forces merge into a single, electroweak force.

Like Maxwell's theory, the standard model is powerful, but there are some details that it can't quite explain. One is dark matter, a so-far undetected entity that makes up most of the matter in the cosmos. Another is dark energy, a force that seems to be pushing the Universe apart. There are some other unexplained odds and ends too, but nothing formidable enough to push the standard model from its perch.

To deal with some of the problems, the best theorists of the day have proposed an extension of the model, known as supersymmetry. This modification unifies the electroweak force with the strong nuclear force, and suggests some elementary particles that might explain dark matter.

Now, an experiment has come along to challenge the standard model and its offspring. The Large Hadron Collider (LHC), a 27-kilometre proton-proton collider on the French-Swiss border near Geneva, Switzerland, is delivering a torrent of data that can be used to probe the boundaries of the standard model. But the collider has yet to find evidence of the particles suggested by supersymmetry theory (see page 13). If it finds nothing in the next year, the theory will look like it is in serious trouble. If it finds nothing in two years, then many theorists will probably abandon it, just as theorists eventually had to abandon extensions of Maxwell's work that explained away the missing ether.

The parallels with history shouldn't be taken too seriously. The LHC is a much more elaborate experiment than the one done by Michelson and Morley. It uses proton collisions to probe unknown energies for all sorts of things, not just the supersymmetrical particles some hope it will find. Nor is the LHC likely to deliver a clear refutation of supersymmetry - the theory, the data and the analysis are all much more complicated than they were 125 years ago.

But comparison can remind us of something that is easily overlooked: the negative results now coming out of the LHC should be just as stimulating as any positive findings. Michelson and Morley's experiment, and others like it, eventually led Albert Einstein to axiomatically accept that light travelled at a constant speed and could be both a wave and a particle. Those revelations never really disproved Maxwell's theories, but they did help to develop special relativity and quantum mechanics - the two greatest theories of the twentieth
DNATURE.COM To comment online, click on Editorials at: go.nature.com/xhunqu century. In the same way, the LHC's results to think differently. If one beautiful theory can't explain the data, then there must be another out there somewhere that can. whatever they may be - could force scientists 